\title{
Hidden dimers and the matrix maps: Fibonacci chains re-visited
}

\author{
Samar Chattopadhyay ${ }^{1}$ and Arunava Chakrabarti ${ }^{2}$ \\ ${ }^{1}$ Department of Physics, Hooghly Mohsin College, Chinsurah, West Bengal 712 101, India. \\ ${ }^{2}$ Department of Physics, University of Kalyani, Kalyani, West Bengal 741 235, India.
}

\begin{abstract}
The existence of cycles of the matrix maps in Fibonacci class of lattices is well established. We show that such cycles are intimately connected with the presence of interesting positional correlations among the constituent 'atoms' in a one dimensional quasiperiodic lattice. We particularly address the transfer model of the classic golden mean Fibonacci chain where a six cycle of the full matrix map exists at the centre of the spectrum [Kohmoto et al, Phys. Rev. B 35, 1020 (1987)], and for which no simple physical picture has so far been provided, to the best of our knowledge. In addition, we show that our prescription leads to a determination of other energy values for a mixed model of the Fibonacci chain, for which the full matrix map may have similar cyclic behaviour. Apart from the standard transfer-model of a golden mean Fibonacci chain, we address a variant of it and the silver mean lattice, where the existence of four cycles of the matrix map is already known to exist. The underlying positional correlations for all such cases are discussed in details.
\end{abstract}

PACS No.s: 71.23.An and 71.23.Ft 
One dimensional quasiperiodic lattices have been the subject of extensive research in the past several years, and we have already gathered a wealth of knowledge about these systems [1-11,13-16, and references therein]. Theoretical studies of the spectral properties of one dimensional quasiperiodic lattices in particular, have occupied a large portion of all these works [see 1-11, for example]. Perhaps the most widely studied model is based on the Fibonacci sequence which started drawing interest after the publication of a couple of pioneering papers by Kohmoto et al [1] and Ostlund et al [2]. The spectral properties of one dimensional Fibonacci chain are exotic. The single particle eigenstates are neither extended, nor localized in a conventional sense, and the spectrum is a Cantor set with zero Lebesgue measure [1-3]. The existence of self similarity and scaling behaviour of the wave functions are two other major properties which mark the difference of such a quasicrystalline chain from a periodic or a randomly disordered lattice. A Fibonacci chain consists of two letters $A$ and $B$. The entire sequence is generated by successive application of the substitution rule, $A \rightarrow A B$ and $B \rightarrow A$. The first few generations are, $G_{0}=A, G_{1}=A B, G_{3}=A B A$, $G_{4}=A B A A B$, and so on, where the letter $G$ indicates 'generation'. The letters $A$ and $B$ may denote two different 'atoms' (site model) or, two different 'bonds' separating identical atoms (transfer model) [7]. The electronic properties of such systems are well described within the standard tight binding formalism, where the Hamiltonian, in the Wannier basis, is given by,

$$
H=\sum_{i} \epsilon_{i}|i\rangle\langle i|+\sum_{i j}\left[t_{i j}|i\rangle\left\langle j\left|+t_{j i}\right| j\right\rangle\langle i|\right]
$$

Here, $\epsilon_{i}$ is the site energy corresponding to the $i$-th site and $t_{i j}=t_{j i}$ is the hopping integral between the $i$-th and the $j$-the sites. To solve for the eigenfunctions it is useful to find the solutions of a set of difference equations which, in the nearest neighbor approximation look like,

$$
\left(E-\epsilon_{i}\right) \psi_{i}=t_{i, i+1} \psi_{i+1}+t_{i, i-1} \psi_{i-1}
$$

Here, $\psi_{i}$ is the amplitude of the wave function on the $i-$ th site. The above equation can equivalently be cast into a matrix form: 


$$
\left(\begin{array}{c}
\psi_{i+1} \\
\psi_{i}
\end{array}\right)=\left(\begin{array}{cc}
\frac{\left(E-\epsilon_{i}\right)}{t_{i, i+1}} & -\frac{t_{i, i-1}}{t_{i, i+1}} \\
1 & 0
\end{array}\right)\left(\begin{array}{c}
\psi_{i} \\
\psi_{i-1}
\end{array}\right)
$$

The matrix,

$$
M_{i}=\left(\begin{array}{cc}
\frac{\left(E-\epsilon_{i}\right)}{t_{i, i+1}} & -\frac{t_{i, i-1}}{t_{i, i+1}} \\
1 & 0
\end{array}\right)
$$

which relates the amplitudes of the wave function at three consecutive sites on the chain, is called the transfer matrix.

For investigating the spectral properties of a quasiperiodic chain, one major approach, using a method of the dynamical systems analysis, has been to generate first a finite segment (a 'rational approximant' $[1,2,7])$ of the Fibonacci chain following the substitution rule. The infinite chain is then constructed by periodically repeating this segment. In this way one can use higher and higher order rational approximants to attain a true quasiperiodic Fibonacci chain in the thermodynamic limit. For any generation $l$ one can construct a transfer matrix $M_{l}$ which is the product of the individual transfer matrices across each atomic site in that $l$-th generation, taken in proper order, i.e., $M_{l}=\prod_{i} M_{i}$. This product matrix $M_{l}$ plays a vital role in determining the character of the spectrum and the eigenfunctions. The allowed eigenvalues for the infinite sequence are extracted from a study of the evolution of the trace of the above transfer matrix at successive generations of the Fibonacci sequence $[1,2,7]$. Two important properties of this product matrix for any generation are now well established. We refer to them with respect to the golden mean Fibonacci chain, which will play a central part in this communication.

(i) The Trace Map: Following the prescription that generates the sequence, it is easy to see that the total transfer matrix for any $l+1$-th generation sequence is related to the matrices corresponding to two previous generations by the relation, $M_{l+1}=M_{l-1} M_{l}$. This leads to a recursion relation between the traces of $M_{n}$, viz,

$$
x_{l+1}=2 x_{l} x_{l-1}-x_{l-2}
$$


Here $x_{l}=\operatorname{Tr}\left(M_{l}\right) / 2$. The "allowed" energy values are those for which $\left|x_{l}\right| \leq 1$. As the generation increases we find that almost all the energy values belong to the escaping orbits of the map [1,2], which speaks for a true singular continuous character of the spectrum.

(ii) The Matrix Map: In addition to the above property, the product matrix $M_{l}$ at any generation $l$ is found to exhibit a cyclic behavior [1-7], viz, $M_{l+m}=M_{l}$ for certain energy eigenvalues that depend on the model considered. To clarify, let us give the example of the so called 'transfer model' of the standard golden mean Fibonacci chain, where the letters $A$ and $B$ stand for two bonds, 'Long' $(L)$ and 'Short' $(S)$ respectively. All the on site potentials are assumed to be same, and put equal to zero. In this model one finds that, at $E=0$ which happens to be the centre of the spectrum in this case, the matrix product $M_{l}$ has a six cycle, viz, $M_{l+6}=M_{l}$ for $l \geq 2$. It may be noticed that such cyclic behaviour of the transfer matrix is also common to quasiperiodic chains other than the golden mean [10]. It is this cyclic property of the matrix $M_{l}$, that has stimulated the present work. The motivation will be clear from the discussion that follows.

The cyclic behaviour of the transfer matrices, as it appears from the previous works in general, has been shown to exist for various quasiperiodic chains without any underlying physical reason. It is not straightforward to prove the existence of cycles of the matrix map for any arbitrary substitutionally generated sequence. Not only that, even if it exists, it is extremely difficult to locate the precise energy eigenvalue for a lattice such as the Fibonacci chain in which the spectrum is a Cantor set having a gap at the vicinity of every energy, at which such a cycle will take place. Precisely, this is the point that motivates us to undertake the present study. We wish to examine whether it is at all possible to provide a simple physical picture that will lead to an understanding the origin of cycles of the matrix map based on the geometric structure of the quasiperiodic chain concerned. In this communication we begin by examining the well known six cycle behaviour at $E=0$ in the simplest transfer model of a golden mean Fibonacci chain. We show that there 
exists hidden dimer- like correlations between the constituent 'atoms'. The dimers are not explicitly displayed, but their presence can be directly linked with the six cycle of the full matrix map at $E=0$. We give a prescription to unravel the other six cycle energy values as well, though, $E=0$ is the only energy for which we have been able to detect a six cycle of the matrix map in the present model. However, in a mixed model of the Fibonacci chain we show that following the same prescription we can evaluate at least two values of the electron energy at which the matrices will repeat after every six generations. Our method is perfectly general, and can be applied to any quasiperiodic sequence. In the latter part of this paper we extend the study to the quasiperiodic silver mean chain [10] to show the presence of similar kinds of dimers and establish their relationship with the four-cycle matrix map at $E=0$ already reported in the literature [10].

In this context it is worth mentioning that the existence of dimers in random and quasiperiodic chains [13-16] has been known to cause unscatterd/ extended eigenstates leading to a perfect transmission. However, the Fibonacci chain and all such lattices in the golden mean class, generated for example, using the rule $A \rightarrow A^{n} B, B \rightarrow A$ [10] have so far remained un-noticed in this respect. In a very recent work on a more involved model of the Fibonacci lattice Satija et al [16] have shown that there exist a single 'resonant' eigenstate that can be explained in terms of the presence of a 'dimer' We begin by studying the oldest and the simplest version of the transfer model of a Fibonacci chain. It will be shown that the dimers in this system exist naturally, but they combine in a nested fashion to give rise to a 'resonance' only when certain conditions are satisfied. In what follows we describe the method of analysis.

The transfer model of a Fibonacci chain comprises of two different 'bonds' $L$ and $S$ generated according to the prescription $L \rightarrow L S$ and $S \rightarrow L$. We attribute three values to the on site potentials, viz, $\epsilon_{\alpha}, \epsilon_{\beta}$ and,$\epsilon_{\gamma}$ corresponding to the sites flanked by $L-L, L-S, S-L$ bonds respectively. The nearest neighbor hopping integrals are designated by $t_{L}$ and $t_{S}$ 
corresponding to the $L$ and $S$ bonds [9]. Let us now define three types of transfer matrices correspondent to the three sites $\alpha, \beta$ and $\gamma$, viz

$$
M_{\alpha}=\left(\begin{array}{cc}
\frac{\left(E-\epsilon_{\alpha}\right)}{t_{L}} & -1 \\
1 & 0
\end{array}\right) ; M_{\beta}=\left(\begin{array}{cc}
\frac{\left(E-\epsilon_{\beta}\right)}{t_{S}} & -\frac{t_{L}}{t_{S}} \\
1 & 0
\end{array}\right) ; M_{\gamma}=\left(\begin{array}{cc}
\frac{\left(E-\epsilon_{\gamma}\right)}{t_{L}} & -\frac{t_{S}}{t_{L}} \\
1 & 0
\end{array}\right)
$$

The transfer matrix for any $l+1$-th generation chain is obtained recursively following the equation,

$$
M_{l+1}=M_{l-1} M_{l}
$$

where, $M_{l}$ is the matrix for the $l$-th generation, obtained by multiplying the above elementary matrices $M_{\alpha}, M_{\beta}$ and $M_{\gamma}$ in proper order. In the above equation, $M_{1}=M_{\alpha}$ and $M_{2}=$ $M_{\gamma} M_{\beta}=M_{\gamma \beta}$ (say) [7]. It has been observed in the literature [7] that in the above model with $\epsilon_{\alpha}=\epsilon_{\beta}=\epsilon_{\gamma}=0$ and $t_{L}=1$ and $t_{S}=2$, one gets $M_{l+6}=M_{l}$ for $l \geq 2$. Let us now give a simple physical picture of this phenomenon based on the existence of the dimer like positional correlations in this model of the Fibonacci chain.

Consider the second generation chain, i.e. $l=2$. This is $L S$. The product transfer matrix, when written explicitly for this segment, is, $M_{2}=M_{\gamma} M_{\beta} M_{\alpha}$. We have taken the first site of this chain to be of type $\alpha$ without the loss of generality. The last site will be of type $\gamma$. This is obvious, because the segment $L S$ has to be repeated periodically, so that the bond appearing after the $S$ will be $L$. The matrix for $l=8$ i.e. $M_{8}$ naturally consists of a much longer string of $M_{\alpha}, M_{\beta}$ and $M_{\gamma}$. Yet, at $E=0$, we all know that $M_{8}=M_{2}$ and this is repeated after every six steps for $l \geq 2$. So, to our mind, a natural question should be, how do the individual matrices combine so that the bigger and bigger strings of matrices shrink back to $M_{2}$ at this particular energy ? This leads us to carefully examine the structure of the Fibonacci chain. In Fig.1 we show a Fibonacci chain in the sixth generation for example. We observe that in it (as well as in an infinite chain), the $\alpha$-sites appear in isolation and the $\beta-\gamma$ sites appear in pair, as well as in isolation. It is important to observe that every isolated $\beta-\gamma$ cluster, as well as $\beta-\gamma$ pairs side by side, are flanked by two $\alpha$-sites. This 
happens everywhere, even in the infinite chain. Now, if the trace of the matrix $M_{\gamma \beta}=M_{\gamma} M_{\beta}$ becomes zero, then the corresponding energy renders the matrix product $M_{\gamma \beta} M_{\gamma \beta}$ identity (barring a negative sign) [12-14]. Once the $\beta-\gamma$ clusters (joined by the solid curve in Fig.1) combine to give us an identity contribution in terms of the matrices, the $\alpha$ sites at the two flanks (joined by a dotted curve in Fig.1) automatically form a 'dimer' [12]. If the trace of $M_{\alpha}$ now vanishes for the same energy, then the product of two consecutive $M_{\alpha}$ matrices also become equal to an identity matrix (once again with a negative sign), so that a local cluster of matrices $M_{\alpha} M_{\gamma \beta} M_{\gamma \beta} M_{\alpha}$ becomes equal to an identity matrix. This will happen locally throughout the chain. In this way one can see that the entire string of matrices will reduce, in 'size', because of the resonance in these two different kinds of dimers $(\alpha-\alpha$ and $\beta \gamma-\beta \gamma)$ simultaneously. These pairs appear in a nested fashion, and appear at all scales of length because of the self-similar character of the lattice. If we get an energy for which $M_{\alpha}^{2}=M_{\gamma \beta}^{2}=-I, I$ being the $2 X 2$ identity matrix, then one can verify that, $M_{l}=M_{l+6}$ for $l \geq 2$. Now, let us see if $E=0$ satisfies this criterion. In the transfer model,

$$
\operatorname{Tr}\left(M_{\gamma \beta}\right)=\frac{\left(E-\epsilon_{\beta}\right)\left(E-\epsilon_{\gamma}\right)-\left(t_{L}^{2}+t_{S}^{2}\right)}{t_{L} t_{S}}
$$

We select $\epsilon_{\alpha}=\epsilon_{\beta}=\epsilon_{\gamma}=0$ and $t_{S}=2$ and $t_{L}=1[7] . E=0$ definitely makes $M_{\alpha}^{2}=-I$, but $\operatorname{Tr}\left(M_{\gamma \beta}\right) \neq 0$ and hence, $M_{\gamma \beta}^{2} \neq I$. The resonance condition discussed above is not satisfied. This of course, does not rule out the possibility of having bigger clusters in the formation of nested dimers. For example, one can see (Fig.2a) that there are clusters formed by the triplet $\beta \gamma \alpha$ and $\beta \gamma$ which are distributed in the same manner and can be considered to be a 'renormalized' version of the basic clusters formed by $\beta \gamma$ and $\alpha$ respectively. It turns out that in the transfer model the clusters responsible for a six cycle of the matrix map at $E=0$ are the pairs $\beta \gamma \alpha \beta \gamma-\beta \gamma \alpha \beta \gamma$ flanked at the two extremities by the cluster $\alpha \beta \gamma$. That is, $\operatorname{Tr}\left(M_{\gamma \beta} M_{\alpha} M_{\gamma \beta}\right)=\operatorname{Tr}\left(M_{\gamma \beta} M_{\alpha}\right)=0$ at $E=0$. Under this condition $M_{l+6}=M_{l}$ for $l \geq 2$. Once this is observed the question that arises immediately is, how to identify the minimal clusters responsible for a possible six (or other) cycle of the full matrix map for the chain. To resolve this issue we get back to the method of real space renormalization scheme, 
applied to the infinite quasiperiodic chains in the literature [9]. It is important however, to appreciate that, the renormalization group method will be used here only to identify the minimal clusters responsible for resonance.

It is known that the on-site potentials and the hopping integrals in the infinite Fibonacci chain renormalize to $[9]$ :

$\epsilon_{\alpha}(n+1)=\epsilon_{\gamma}(n)+\left[t_{L}(n)^{2}+t_{S}(n)^{2}\right] /\left(E-\epsilon_{\beta}(n)\right) ; \epsilon_{\beta}(n+1)=\epsilon_{\gamma}(n)+t_{S}(n)^{2} /\left(E-\epsilon_{\beta}(n)\right)$

$\epsilon_{\gamma}(n+1)^{2}=\epsilon_{\alpha}(n)+t_{L}(n)^{2} /\left(E-\epsilon_{\beta}(n)\right) ; t_{L}(n+1)=t_{L}(n) t_{S}(n) /\left(E-\epsilon_{\beta}(n)\right)$ and $t_{S}(n+1)=$ $t_{L}(n)$.

One defines a parameter space of reduced dimensionality, and defined by the dimensionless quantities $w, x, y$ and $z$, where,

$$
w_{n}=\left(E-\epsilon_{\alpha}(n)\right) / t_{L}(n), x_{n}=\left(E-\epsilon_{\beta}(n)\right) / t_{L}(n), y_{n}=\left(E-\epsilon_{\gamma}(n)\right) / t_{L}(n) \text { and, } z_{n}=
$$
$t_{S}(n) / t_{L}(n)$. These parameters, under renormalization give rise to the following set of recursion relations [9],

$w_{n+1}=\left(x_{n} y_{n}\right) / z_{n}-z_{n}-1 / z_{n} ; x_{n+1}=\left(x_{n} y_{n}\right) / z_{n}-z_{n} ; y_{n+1}=\left(w_{n} x_{n}\right) / z_{n}-1 / z_{n}$, and, $z_{n+1}=x_{n} / z_{n}$.

From the above recursion relations one can show that

$$
w_{n+3}=w_{n+2} w_{n+1}-w_{n}
$$

which is equivalent to the trace map (3) with $w_{n}=2 x_{n}$. Using the set of recursion relations (7) together with Eq.(6) it is straightforward to show that if we set $w_{n}=w_{n+1}=0$ then (i) $w_{n+3}=w_{n}$, and (ii) $\left(w_{n+6}, x_{n+6}, y_{n+6}, z_{n+6}\right)=\left(w_{n}, x_{n}, y_{n}, z_{n}\right)$. That is, the entire parameter space is mapped onto itself after six steps of renormalization. Additionally, one comes across a three cycle of the trace map (as $w_{n+3}=w_{n}$ ). As the total transfer matrix across any arbitrary generation can be completely expressed in terms of the parameters $w, x, y$ and $z$, it is obvious that the transfer matrix for the generation $n+6$ will be identical to that in the 
generation $n$ for the energy value extracted by setting $w_{n}=w_{n+1}=0$. Most interestingly, it can be checked that $w_{n}$ is actually $\operatorname{Tr}\left(M_{\alpha, n}\right)$ and $w_{n+1}=\operatorname{Tr}\left(M_{\alpha, n+1}\right)=\operatorname{Tr}\left(M_{\gamma \beta, n}\right)$, where $M_{i, n}$ is the transfer matrix for the $i$-th site in the $n$ - step renormalized lattice. Therefore, setting $w_{n}=w_{n+1}=0$ is equivalent to searching for simultaneous zeros for the polynomial equations $\operatorname{Tr}\left(M_{\alpha, n}\right)=0$ and $\operatorname{Tr}\left(M_{\gamma \beta, n}\right)=0$. If we start with an infinite Fibonacci chain and re-scale it, we observe that the resonance condition, as described above, is satisfied for the first time on a two times renormalized version of the original Fibonacci chain (i.e. $n=2$ ) if we take $E=0$. That is, for $E=0$, we get $w_{2}=0$ and $w_{3}=0$ simultaneously. The $\alpha-\alpha$ and the $\gamma \beta-\gamma \beta$ pairs at this length scale form the required 'dimers' causing resonance. To see what minimal clusters do they correspond to in terms of the original chain, we trace back to the lattice at the original length scale to see that the elementary blocks causing resonance are clusters like $\alpha \beta \gamma$ and $\beta \gamma \alpha \beta \gamma$ respectively. The 'dimers' in a one-step renormalized lattice and their correspondence with the 'dimers' in the original, un-renormalized lattice is illustrated in Fig.2. Doing one more step is simple. It should be noted that the method outlined here indicates the possible existence of six cycle for other values of $E$. For example, one could search for simultaneous zero's of, say, $w_{4}$ and $w_{5}$ etc. However, we have been unable to detect any other value for the energy in this model that may lead to other cyclic behaviors. But, we propose a second model which clearly shows that based on the same thread of reasoning one can get more than one energy values for which a six cycle map for the transfer matrix exists.

\section{A mixed model:}

Let us select a model in which $\epsilon_{\alpha}=\sqrt{5}, \epsilon_{\beta}=\epsilon_{\gamma}=0, t_{L}=1$ and $t_{S}=2$. With this choice of the parameters we find that, $\operatorname{Tr}\left(M_{\alpha}\right)=\operatorname{Tr}\left(M_{\gamma \beta}\right)=0$ in the original (un-renormalized) lattice if we set $E=\sqrt{5}$. This leads to the fact that if we start with the above mixed model Fibonacci chain at the 8-th generation, then the $\alpha-\alpha$ and $\beta \gamma-\beta \gamma$ pairs in the original lattice will form the nested dimers. The corresponding transfer matrices will combine together to 
give rise to an identity matrix, and one is finally left with a product of $M_{\gamma \beta} M_{\alpha}$ in the 8 -th generation corresponding to the triplet of sites $\alpha \beta \gamma$ which is the same as the second generation chain. That is, we achieve the result $M_{8}=M_{2}, M_{9}=M_{3}$ and so on. Let us now renormalize the lattice once and look for a simultaneous solution of the equations $w_{2}=0$ and $w_{3}=0$. Most interestingly we find that $w_{2}=w_{3}=0$ for $E=-\sqrt{5}$. If we trace back to the original lattice it is easy to discern that the fundamental clusters responsible for

resonance in this case are $\alpha \beta \gamma$ and $\beta \gamma$ respectively. This implies that for $E=-\sqrt{5}$ the six cycle feature $M_{l}=M_{l+6}$ sets in for $l \geq 3$. Thus we have been able to identify two different energy eigenvalues for this mixed model of the Fibonacci chain for which the matrix map has a six cycle. For the two different energy eigenvalues the 'dimer correlations' are revealed at two different scales of length. This is an important feature, linked with the inherent self similarity of the lattice concerned.

\section{The silver mean lattice:}

To check the validity of our arguments further, we have extended this idea to the transfer model of the silver mean lattice, which is generated according to the rule $L \rightarrow L L S$ and $S \rightarrow L$. It may be mentioned at this point, that the entire discussion made for the silver mean case is valid, in general, for the class of lattices grown according to the rule $L \rightarrow L^{m} S$, $S \rightarrow L$. The recursion relations depicting the parameter space in the silver mean case are $[17]$

$w_{n+1}=\left(w_{n} x_{n}-1\right) y_{n} / z_{n}-w_{n} z_{n}-x_{n} / z_{n} ; x_{n+1}=\left(w_{n} x_{n}-1\right) y_{n} / z_{n}-w_{n} z_{n} ; y_{n+1}=\left(w_{n} x_{n}-\right.$ 1) $w_{n} / z_{n}-x_{n} / z_{n} ; z_{n+1}=\left(w_{n} x_{n}-1\right) / z_{n}$, and, $t_{n+1}=x_{n} y_{n} / z_{n}-z_{n}-1 / z_{n}$

From the above relations it follows that [17], $w_{n+2}=w_{n+1} t_{n+2}-w_{n}$ and, $t_{n+2}=w_{n} w_{n+1}-$ $t_{n+1}$. The basic clusters responsible for dimer correlation in this case are $\alpha-\alpha$ and $\alpha \beta \gamma-\alpha \beta \gamma$ respectively. We find that, $\left(w_{n+4}, x_{n+4}, y_{n+4}, z_{n+4}\right)=\left(w_{n}, x_{n}, y_{n}, z_{n}\right)$ if we set $w_{n}=w_{n+1}=0$. This happens for $E=0$. That means the four cycle of the matrix map [10] is observed following our prescription. The trace map exhibits a two cycle behavior in this case, as 
$w_{n+2}=w_{n}$. One can look for other possible cycles of $M_{l}$ by renormalizing the lattice and following the arguments given earlier. These aspects are now being investigated and the results will be published in due course.

\section{Acknowledgment:}

The authors are thankful to R. K. Moitra and S. N. Karmakar for suggestions. One of the authors (A.C.) acknowledges interesting discussions with Michael Schreiber. 


\section{References}

[1] M. Kohomoto, L. P. Kadanoff and C. Tang, Phys. Rev. Lett. 50, 1870 (1983).

[2] S. Ostlund et al., Phys. Rev. Lett. 50, 1873 (1983).

[3] M. Kohomoto and Y. Oono, Phys. Lett. A 102, 145 (1984); M. Kohmoto and J. R. Banavar, Phys. Rev. B 34, 563 (1986); J. P. Lu, T. Odagaki and J. L. Birman, Phys. Rev. B 33, 4809 (1986); F. Nori and J. P. Rodriguez, Phys. Rev. B 34, 2207 (1986).

[4] B. Simon, Adv. Appl. Math. 3, 463 (1982); J. B. Sokoloff, Phys. Rep. 126, 189 (1985);

J. M. Luck and D. Petritis, J. Stat. Phys. 42, 289 (1986).

[5]. C. Tang and M. Kohmoto, Phys. Rev. B 34, 2041 (1986); B. Sutherland, Phys. Rev. Lett. 57, 770 (1986);

[6] F. Deylon and D. Petritis, Commun. Math. Phys. 103, 441 (1986); Q. Niu and F. Nori, Phys. Rev. Lett. 57, 2057 (1986).

[7] M. Kohmoto, B. Sutherland and C. Tang, Phys. Rev. B 35, 1020 (1987).

[8] Y. Liu and R. Riklund, Phys. Rev. B 35, 6034 (1987); R. Riklund, M. Severin and Y. Liu, Int. J. Mod. Phys. B 1, 121 (1987).

[9] J. A. Ashraff and R. B. Stinchcombe, Phys. Rev. B 37, 5723 (1988).

[10] G. Gumbs and M. K. Ali, Phys. Rev. Lett. 60, 1081 (1988); G. Gumbs and M. K. Ali, J. Phys. A:Math. Gen. 22, 951 (1989); Q. Niu and F. Nori, Phys. Rev. B 42, 10329 (1990); J. M. Luck, Phys. Rev. B 39, 5834 (1989); A. Bovier, J. Phys. A: Math.Gen. 25, 1021 (1992); A. Bovier and J. M. Ghes, Commun. Math. Phys. 15845 (1993).

[11] A. Sanchez, E. Macia and F. Dominguez-Adame, Phys. Rev. B 49, 147 (1994); E. Macia and F. Dominguez-Adame, Phys. Rev. Lett. 76, 2957 (1996); ibid 79, 5301 (1997).

[12] E. Macia and F. Dominguez-Adame, in Electrons, Phonons and Excitons in low dimensional aperiodic systems, and references therein, Editorial Coplutense, Madrid (2000).

[13] D. H. Dunlap, H-L. Wu and P. W. Phillips, Phys. Rev. Lett. 65, 88 (1990).

[14] Arunava Chakrabarti, S. N. Karmakar and R. K. Moitra, Phys. Rev. B 50, 13276 (1994). 
[15] Arunava Chakrabarti, S. N. Karmakar and R. K. Moitra, Phys. Rev. Lett. 74, 1403 (1995).

[16] I. G. Guesta and Indubala Satija, cond-mat/9904029, April 1 (1999).

[17] Arunava Chakrabarti and S. N. Karmakar, Phys. Rev. B 44, 896 (1991). 


\section{Figure Captions:}

Fig.1: Sixth generation Fibonacci chain with three different sites. Clusters forming dimers are displayed by solid (for $\beta-\gamma$ ) and dashed (for $\alpha$ ) lines respectively.

Fig.2: Portion of an infinite Fibonacci chain. Clusters responsible for dimer-like correlations in one-step renormalized lattice (b) are the $\beta-\gamma$ doublet (dashed box) and the single $\alpha$ sites (solid box). They correspond to the triplet $\beta \gamma \alpha$ (dashed box) and the doublet $\beta \gamma$ (solid box) respectively,in the un-renormalized lattice (a). 


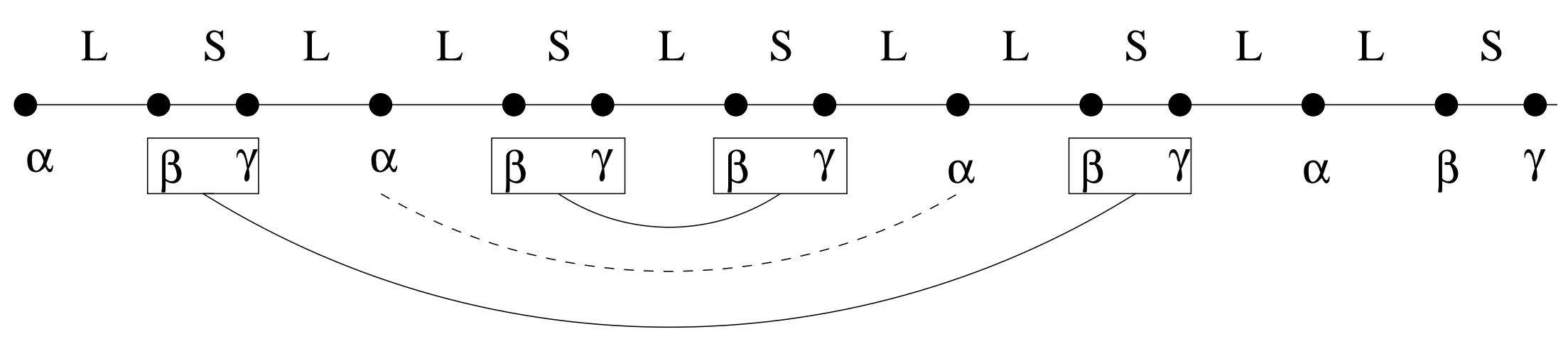

Fig. 1 


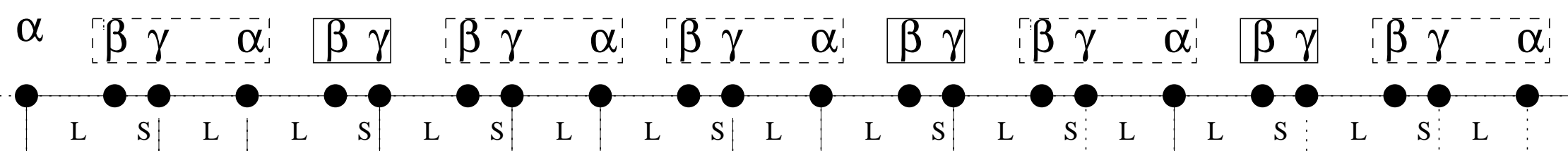

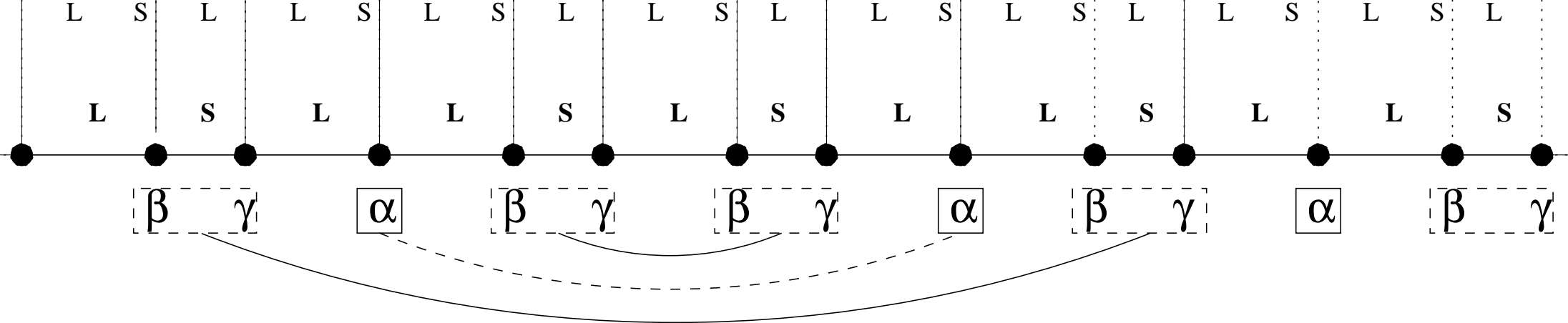

Fig. 2 